\title{
EL GÉNERO Actinostroma NICHOLSON (ESTROMATOPOROIDEOS) EN LA FORMACIÓN SANTA LUCÍA (DEVÓNICO, CORDILLERA CANTÁBRICA, NO DE ESPAÑA)
}

\author{
Isabel MÉNDEZ-BEDIA \\ Departamento de Geología, Universidad de Oviedo. C/ Jesús Arias de Velasco \\ s/n. 33005 Oviedo, España.
}

\begin{abstract}
Méndez-Bedia, I. 1999. El género Actinostroma Nicholson (estromatoporoideos) en la Formación Santa Lucía (Devónico, Cordillera Cantábrica, NO de España). [The genus Actinostroma Nicholson (stromatoporoids) from the Santa Lucía Formation (Devonian, Cantabrian Mountains, NW Spain)]. Revista Española de Paleontología, $n^{0}$ extr. homenaje al Prof. J. Truyols, 121-128. ISSN 0213-6937.
\end{abstract}

\begin{abstract}
The Santa Lucía Formation, from the Devonian (late Emsian-early Eifelian) in the Cantabrian Mountains (NW Spain), yields an abundant and diversified stromatoporoid fauna; one of the most common taxa is the genus Actinostroma Nicholson. In this work the specimens studied are assigned to four species of this genus, $A$. stellulatum Nicholson, A. verrucosum (Goldfuss), A. geminatum Lecompte and $A$. cf. conglomeratum Lecompte. The last two species have already been reported from the Cantabrian Mountains, but they are figured for the first time. Actinostroma verrucosum, A. geminatum and A. cf. conglomeratum, corresponding to the Actinostroma verrucosum group of Lecompte, have been a matter of discussion and, even, assigned to the genus Nexililamina Mallett. The above mentioned species show a typical hexactinellid network which allows us to keep them in the genus Actinostroma s. l.
\end{abstract}

Keywords: Stromatoporoids, Actinostroma, Devonian, Cantabrian Mountains, NW Spain.

\section{RESUMEN}

La Formación Santa Lucía, del Devónico (Emsiense superior-Eifeliense) de la Cordillera Cantábrica (NO de España), presenta una abundante y diversa fauna de estromatoporoideos; uno de los taxones más comunes es el género Actinostroma Nicholson. En este trabajo se asignan los ejemplares estudiados a cuatro especies del género, Actinostroma stellulatum Nicholson, A. verrucosum (Goldfuss), A. geminatum Lecompte y A. cf. conglomeratum Lecompte. Las dos últimas especies han sido ya citadas en la Cordillera Cantábrica, pero se figuran por primera vez. Actinostroma verrucosum, A. geminatum y A. cf. conglomeratum, correspondientes al grupo de Actinostroma verrucosum de Lecompte, han sido objeto de discusión e, incluso, se ha propuesto su asignación al género Nexililamina Mallett. Las especies objeto de este trabajo presentan todas en sección tangencial una malla hexactinélida característica, que permite mantener su asignación al género Actinostroma s.l.

Palabras clave: Estromatoporoideos, Actinostroma, Devónico, Cordillera Cantábrica, NO de España.

\section{INTRODUCCIÓN}

El Devónico con facies Astur-leonesa (Brouwer, 1964) de la Cordillera Cantábrica (NO de España) está constituido por una alternancia de formaciones siliciclásticas y carbonatadas, básicamente con fauna bentónica y depositadas, por lo general, en condiciones marinas someras (Fig. 1A).

En el marco de esta facies se desarrolla una unidad litoestratigráfica esencialmente calcárea, la Formación
Santa Lucía (Emsiense superior-Eifeliense), que presenta una diversa y abundante fauna de estromatoporoideos. Este grupo de organismos fósiles, junto con corales, jugaron un papel muy importante en el desarrollo de numerosos y variados tipos de bioconstrucciones (Méndez-Bedia et al., 1994).

El estudio sistemático de un gran número de ejemplares de estromatoporoideos, recogidos en diversas localidades en las que aflora la Formación Santa Lucía, ha puesto de manifiesto que uno de los taxones más 


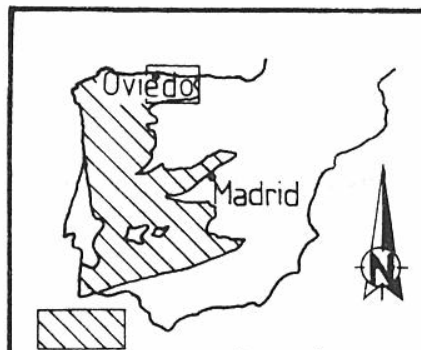

Paleozoico y Precámbrico del Macizo lbérico
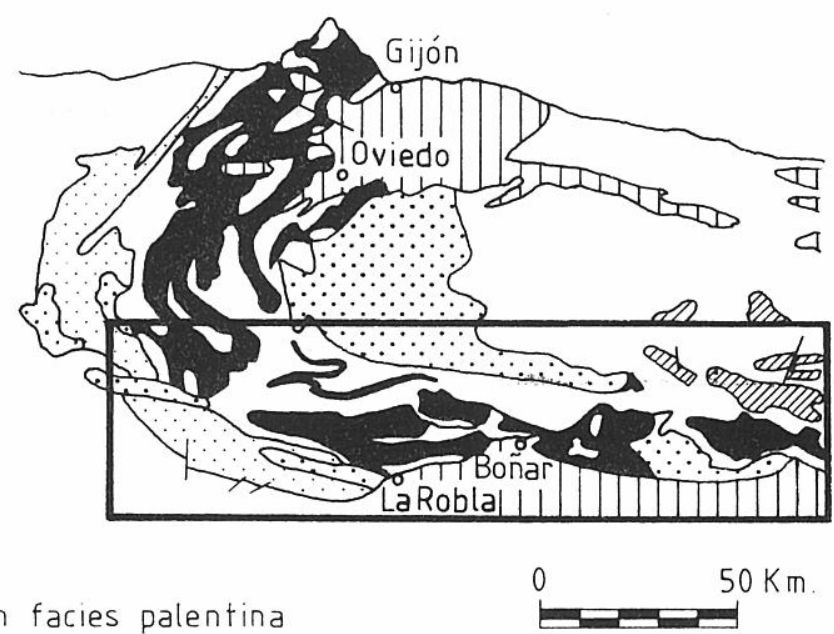

$\because \because \because$ Carbonífero $\square$ Devónico con facies astur-leonesa $\square$ Paleozoico indiferenciado

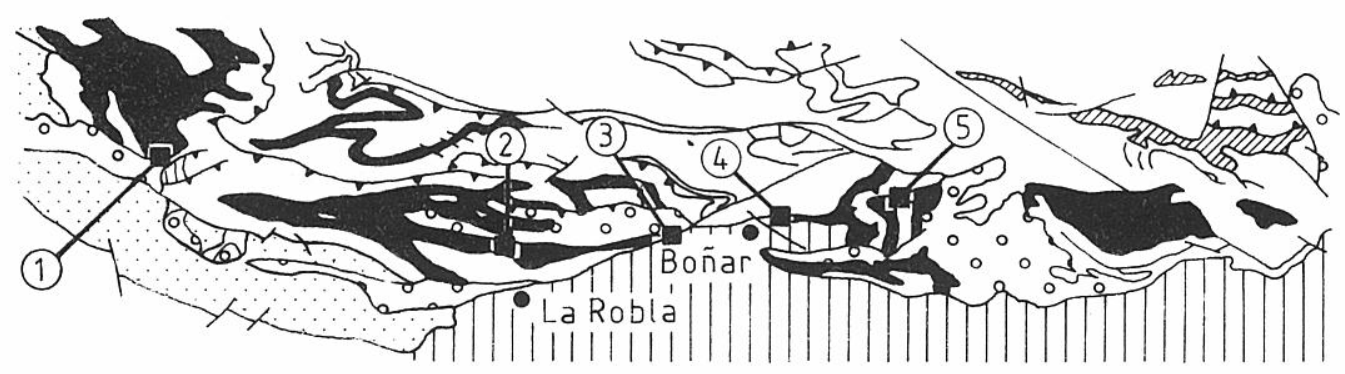

0

$30 \mathrm{Km}$
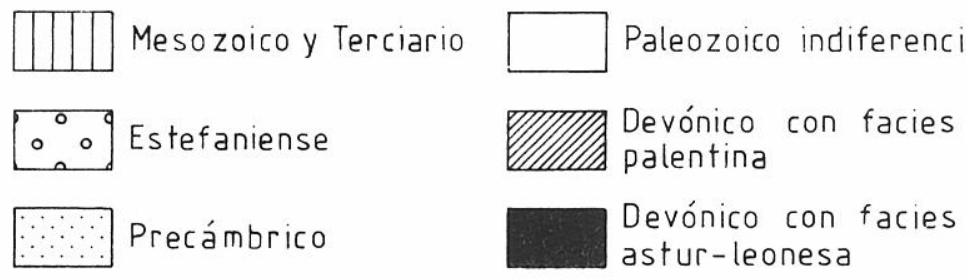

Localidades estudiadas

[0. Estefaniense

$\because \because$ Precámbrico

astur-leonesa
ـ Traza de superficies de cabalgamiento

- Falla

Figura 1. Esquema geológico de la Zona Cantábrica, en el que se muestra: A, distribución de los afloramientos devónicos con facies astur-leonesa y facies palentina, y localización del área estudiada. B, situación de las secciones estudiadas. 1: La Vega de los Viejos; 2: El Millar; 3: Aviados; 4: Adrados; 5: Argovejo.

abundantes en esta formación es el género Actinostroma Nicholson, 1886b, el cual, junto con Stromatoporella Nicholson, 1886b, son los más comunes (Méndez-Bedia y Mistiaen, 1997).

El objeto del presente trabajo consiste en dar a conocer parte de los estudios realizados hasta el presente, en relación con las especies de estromatoporoideos pertenecientes al género Actinostroma. A partir del análisis en lámina delgada de las características internas de los ejemplares, se han identificado cuatro especies, sobre las que se realizarán, en los apartados siguientes, los oportunos comentarios. No obstante, cabe destacar que el género, en la Formacion Santa Lucía, presenta una gran diversidad; pero una buena parte de los ejemplares que pertenecen indudablemente al género, se han mantenido, por el momento, en nomenclatura abierta y serán motivo de un estudio posterior, que permita discriminar de manera más adecuada los caracteres específicos.

Para el estudio realizado en este trabajo se han seleccionado alrededor de cincuenta ejemplares procedentes de cinco secciones de la Formación Santa Lucía (Fig. 1B), localizadas en la vertiente $\mathrm{S}$ de la Cordillera Cantábrica (La Vega de los Viejos, El Millar, 
Aviados, Adrados y Argovejo), en donde se encuentran constituyendo depósitos biostromales y biohermales de edad Emsiense superior. De acuerdo con Méndez-Bedia (1976) las diferentes facies carbonatadas de la Formación Santa Lucía se disponen en franjas paralelas dibujando el Arco Asturiano, con facies sublitorales hacia el S (zonas externas de la plataforma carbonatada) y facies más someras, depósitos lagunales y perilitorales, hacia el $\mathrm{N}$ (zonas internas de la plataforma). Entre ambos tipos de facies se desarrollan facies intermedias con características sublitorales y perilitorales.

Los ejemplares estudiados, junto con las láminas delgadas se hallan depositados en el Departamento de Geología de la Universidad de Oviedo con las siglas DPO.

\section{ANTECEDENTES HISTÓRICOS SOBRE EL GÉNERO Actinostroma NICHOLSON, 1886 EN LA FORMACIÓN SANTA LUCÍA}

A lo largo de sus campañas de trabajo en la Cordillera Cantábrica, Barrois recogió muestras de estromatoporoideos, las cuales confió a Bargatzky. Los resultados de este estudio fueron presentados por el propio Barrois en una reunion de la Société Géologique du Nord, que tuvo lugar en mayo de 1882, e incluidos en su gran monografía (Barrois, 1882). Bargatzky (1883), en una breve nota, describió sin figuraciones dos especies de estromatoporoideos. Una de ellas, Stromatopora concentrica Goldfuss, 1826 procedía de la Formación Moniello, equivalente de la Formación Santa Lucía en la vertiente $\mathrm{N}$ de la Cordillera Cantábrica. No obstante, revisiones realizadas por Nicholson $(1889,1891)$ y Lecompte (1951) sobre material original de Bargatzky (1881), determinaron que la especie descrita por este autor era incuestionablemente sinónima de Actinostroma clathratum Nicholson, 1886a. Sería necesario revisar el material cantábrico descrito por Bargatzky, cuyo paradero se desconoce, para poder emitir una opinión sobre su pertenencia al género Actinostroma Nicholson, 1886b.

Sleumer (1969) describió y figuró seis especies del género Actinostroma, procedentes de diversas localidades en las que aflora la Formación Santa Lucía; dos de estas localidades, La Vega de los Viejos y Argovejo, son las mismas de las que procede parte del material objeto de este trabajo. De las seis especies, cuatro son mantenidas en nomenclatura abierta, Actinostroma sp. 1, Actinostroma sp. 2, Actinostroma sp. 3 y Actinostroma sp. 4, y las otras dos atribuidas a A. papillosum (Bargatzky, 1881) y A. verrucosum (Goldfuss, 1826)?

Méndez-Bedia (1984) figuró y realizó una serie de observaciones sobre los estromatoporoideos de la Formación Moniello (vertiente N de la Cordillera Cantábrica), en la localidad de Arnao (Asturias). Por lo que se refiere al género Actinostroma, se incluyeron varios comentarios sobre Actinostroma verrucosum (Goldfuss) y A. cf. stellulatum Nicholson. Méndez-Bedia, en Soto y Méndez-Bedia (1985), cita de la misma formación y localidad una forma de estromatoporoideo, atribuida al género Actinostroma.

Méndez-Bedia, en Truyols et al. (1990), sintetiza muy resumidamente el desarrollo arrecifal en la Cordillera Cantábrica y recoge las citas de las diferentes especies de estromatoporoideos conocidas hasta el momento; entre ellas se hace mención a diferentes especies de Actinostroma.

Por último, Méndez-Bedia et al. (1994) realizan un detallado estudio sobre los episodios arrecifales que tuvieron lugar durante el Devónico en la Cordillera Cantábrica y, basándose en los constituyentes paleontológicos y facies relacionadas, establecen y describen numerosos tipos de bioconstrucciones. A modo de apéndice, se suministra una lista de los taxones más importantes que contribuyen a la formación de los diferentes tipos de estructuras arrecifales. Entre otros estromatoporoideos, se citan en la Formación Santa Lucía varias especies del género Actinostroma: Actinostroma geminatum Lecompte, 1951; A. stellulatum Nicholson, 1886a; A. verrucosum (Goldfuss, 1826) y Actinostroma sp.

\section{CONSIDERACIONES Y COMENTARIOS PALEONTOLÓGICOS}

El género Actinostroma es uno de los taxones de estromatoporoideos más abundantes y cosmopolitas durante el Devónico. Se caracteriza por presentar los elementos esqueléticos bien diferenciados y perpendiculares entre sí; pilares continuos a través de más de un espacio interlaminar y láminas reducidas a una especie de procesos o prolongaciones horizontales (colliculi), que surgen radialmente de los pilares a ciertos intervalos. Estos procesos se unen entre ellos dando como resultado, en sección tangencial, una malla muy característica que ha sido denominada malla hexactinélida. La microstructura es compacta o esponjosa, presentando los pilares, a veces, un eje oscuro o, más raramente, una zona axial clara. Stearn (1966, p. $87 ; 1980$, p. 894) indica que la malla hexactinélida en corte tangencial es el sine qua non del género Actinostroma.

Flügel (1959) hizo una profunda revisión del género, teniendo en cuenta todas las especies descritas hasta esa fecha, situándolas en ocho grupos dentro de dos subgéneros. Stearn (1966, p. 87) realizó una discusión sobre Actinostroma e indicó claramente las diferencias existentes con géneros próximos. Stock (1982, p. 669) presentó una sinonimia muy completa del género, así como la comparación con géneros vecinos.

El género Actinostroma está representado en la Formación Santa Lucía, entre otras especies, por $A$. stellulatum, A. verrucosum, A. geminatum y $A$. cf. conglomeratum. La mayor parte de estas formas ( $A$. verrucosum, A. geminatum y $A$. cf. conglomeratum) pertenecen al grupo de Actinostroma verrucosum propuesto por Lecompte (1951) y han sido objeto de discusión por parte de varios autores. Así Mallett (1971) 
creó el nuevo género Nexililamina, cuya especie-tipo es N. dipcreekensis Mallett, 1971, e incluyó dentro de este género las especies del grupo A. verrucosum de Lecompte (1951) y especies similares posteriormente descritas. Este género, según su autor, está muy próximo a Actinostroma y sólo se diferencia de él por la posesión de láminas complejas, ausencia de colliculi o prolongaciones radiales y por pilares frecuentemente subdivididos en su contacto con las láminas. La discutida diagnosis de Nexililamina ha sido enmendada recientemente por Webby y Zhen (1997) y el taxón ha sido considerado por estos autores como un género válido. Se caracteriza por láminas compactas, pilares de dos tipos (unos cortos y en forma de V y otros largos y superpuestos) que, en corte tangencial, presentan secciones redondeadas a irregulares y microstructura celular. El género carece, pues, entre otras características, de la típica malla hexactinélida, que en opinión de Stearn (1966) es uno de los rasgos esenciales en Actinostroma.

Las especies de estromatoporoideos objeto de este trabajo poseen una serie de características que las separan del género Nexililamina Mallett, como son la presencia en todas ellas de una clara malla hexactinélida en corte tangencial, pilares continuos, ausencia de pilares en forma de $\mathrm{V}$ y de microstructura celular, por lo que consecuentemente deben mantenerse dentro del género Actinostroma s.l. Así mismo, Stearn (1991, p. 616) indica que todas las especies del grupo de Actinostroma verrucosum, excepto $A$. ingens Ünsalaner, 1951, tienen pilares largos o superpuestos y que genéricamente están más próximas a Actinostroma que a otros géneros relacionados (Nexililamina Mallett, 1971; Schistodictyon Lesovaya, 1970; Gerronostroma Yavorsky, 1931...).

En lo que sigue se realizan una serie de comentarios sobre cada una de las especies de Actinostroma que están mejor representadas y más ampliamente distribuidas en la Formación Santa Lucía (vertiente S de la Cordillera Cantábrica). En Méndez-Bedia et al (1994) se indica la posición precisa de los niveles de los que procede el material estudiado.

Actinostroma stellulatum Nicholson, 1886a (Fig. 2A, B), está representada por 16 ejemplares con formas de crecimiento variables, desde laminares y tabulares a hemisféricas y subesféricas. Pueden alcanzar $60 \mathrm{~cm}$ de largo y $50 \mathrm{~cm}$ de alto y, en ocasiones, presentan latilaminación y mamelones visibles. Los ejemplares presentan las características estructurales típicas del género Actinostroma con pilares continuos y malla hexactinélida. Los especímenes estudiados son formas muy próximas a Actinostroma stellulatum, tanto por el espesor y densidad de los elementos esqueléticos, como por la presencia de astrorrizas. En sección vertical tienen, por término medio, unas 25 a 32 láminas en $5 \mathrm{~mm}$, con espesores de $0,025 \mathrm{~mm}$ a $0,045 \mathrm{~mm}$, y unos 26 a 33 pilares, con espesores de 0,045 a $0,08 \mathrm{~mm}$. Algunos especímenes han sido recolectados en la parte inferior de la Formación Santa Lucía, en la localidad de La Vega de los Viejos (niveles VV-1, VV-2 y VV-3); otros, en la parte superior de la formación, en la localidad de Argovejo (AR-4a y AR-4c) y, finalmente, otros ejemplares proceden de la parte inferior y superior de la formación, en la localidad de Aviados (AV-1 y AV-4). Esta especie está ampliamente representada en el Dominio del Viejo Mundo. Su distribución geográfica y estratigráfica es la siguiente: España y Australia: Devónico Inferior, Emsiense superior; España, Alemania, Inglaterra, Bélgica, Austria, Polonia, República Checa, Francia, antigua Unión Soviética, S de China y Australia: Devónico Medio, Eifeliense-Givetiense; Alemania y Polonia: Devónico Superior, Frasniense inferior.

De Actinostroma verrucosum (Goldfuss, 1826) (Fig. 2C-E) se poseen 10 ejemplares, en su mayoría, constituidos por fragmentos de morfología subesférica, aunque también algunos tienen morfología nodular y tabular, con ondulaciones frecuentes y mamelones, a veces visibles en superficie. Estos ejemplares pertenecen indudablemente al género Actinostroma ya que en sección tangencial presentan una malla hexactinélida característica. Los ejemplares estudiados son formas muy próximas a Actinostroma verrucosum. Presentan láminas con ondulaciones, de aspecto continuo, con límite superior bien marcado e inferior menos neto e irregular, mostrando frecuentemente en esta parte inferior una zona con disepimentos. La densidad de los elementos esqueléticos coincide bastante con los valores suministrados por Lecompte (1951) para la especie-tipo; sólo los espesores de láminas y pilares son ligeramente menores. Los ejemplares de la Formación Santa Lucía presentan una media de 19 a 26 láminas en $5 \mathrm{~mm}$, con espesores de 0,03 a $0,05 \mathrm{~mm}$, a veces $0,08 \mathrm{~mm}$, y 20 a 22 pilares, más raramente 25 , con espesores de 0,04 a 0,12 $\mathrm{mm}$. Los ejemplares provienen de la parte inferior de la formación, en las localidades de La Vega de los Viejos (VV-1) y Adrados (ADR-1), y de la parte inferior y superior de la misma, en la localidad de Aviados (AV-1, AV-2a y AV-3"). Esta especie es prácticamente cosmopolita en el Dominio del Viejo Mundo. Se encuentra en España: Devónico Inferior, Emsiense superior; Alemania, Inglaterra, Francia, Bélgica,

Figura 2. A, B. Actinostroma stellulatum Nicholson, 1886a. A, sección vertical del ejemplar DPO 33539 en la que se observan astrorrizas superpuestas. x 10. B, sección tangencial del mismo ejemplar en la que se muestra una malla hexactinélida. x 10. C-E. Actinostroma verrucosum (Goldfuss, 1826). C, sección vertical del ejemplar DPO 33540 en la que se observan láminas onduladas con límites superiores bien marcados e inferiores menos netos, a veces, con una zona de disepimentos. x 5. D, sección vertical del ejemplar DPO 33541. x 10. E, sección tangencial del ejemplar DPO 33540 en la que se muestra una malla hexactinélida. x 5. F-H. Actinostroma geminatum Lecompte, 1951. F, sección vertical del ejemplar DPO 33542 en la que se muestran láminas agrupadas. x 5. G, detalle de la sección anterior. x 10. H, sección tangencial del mismo ejemplar en la que se observa, en la parte inferior de la fotografía, una malla hexactinélida y secciones circulares de pilares. x 10 . 

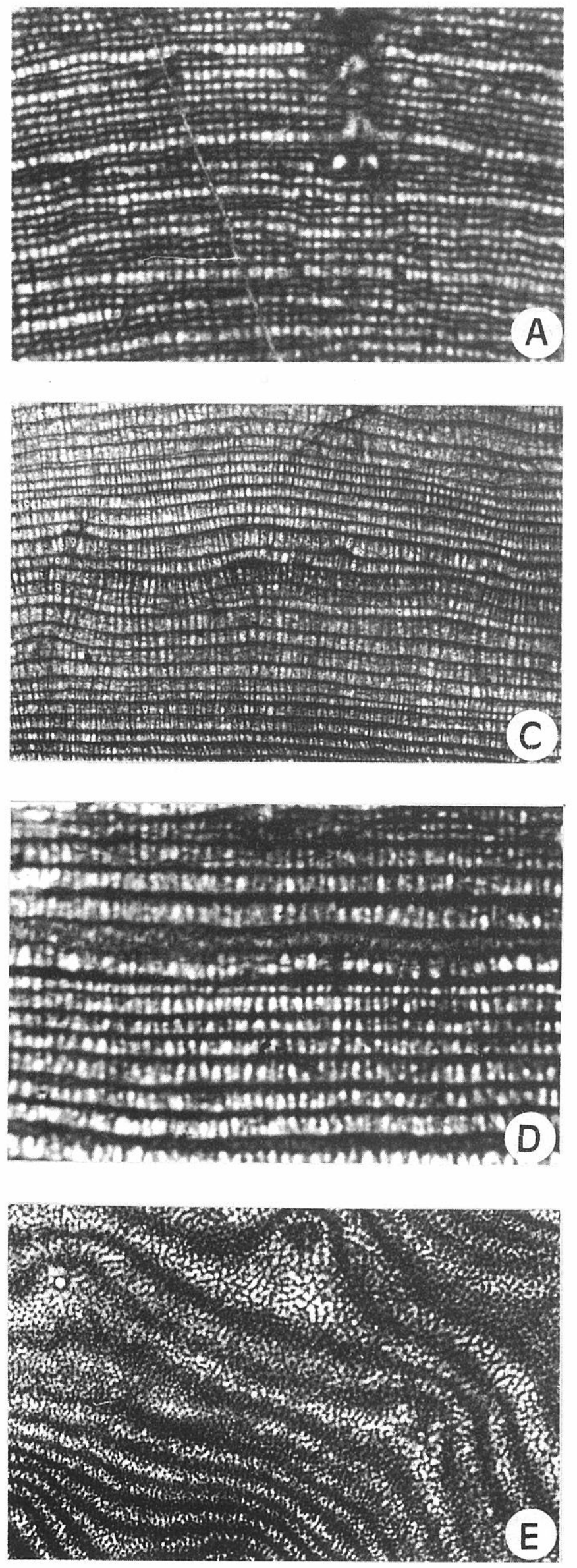
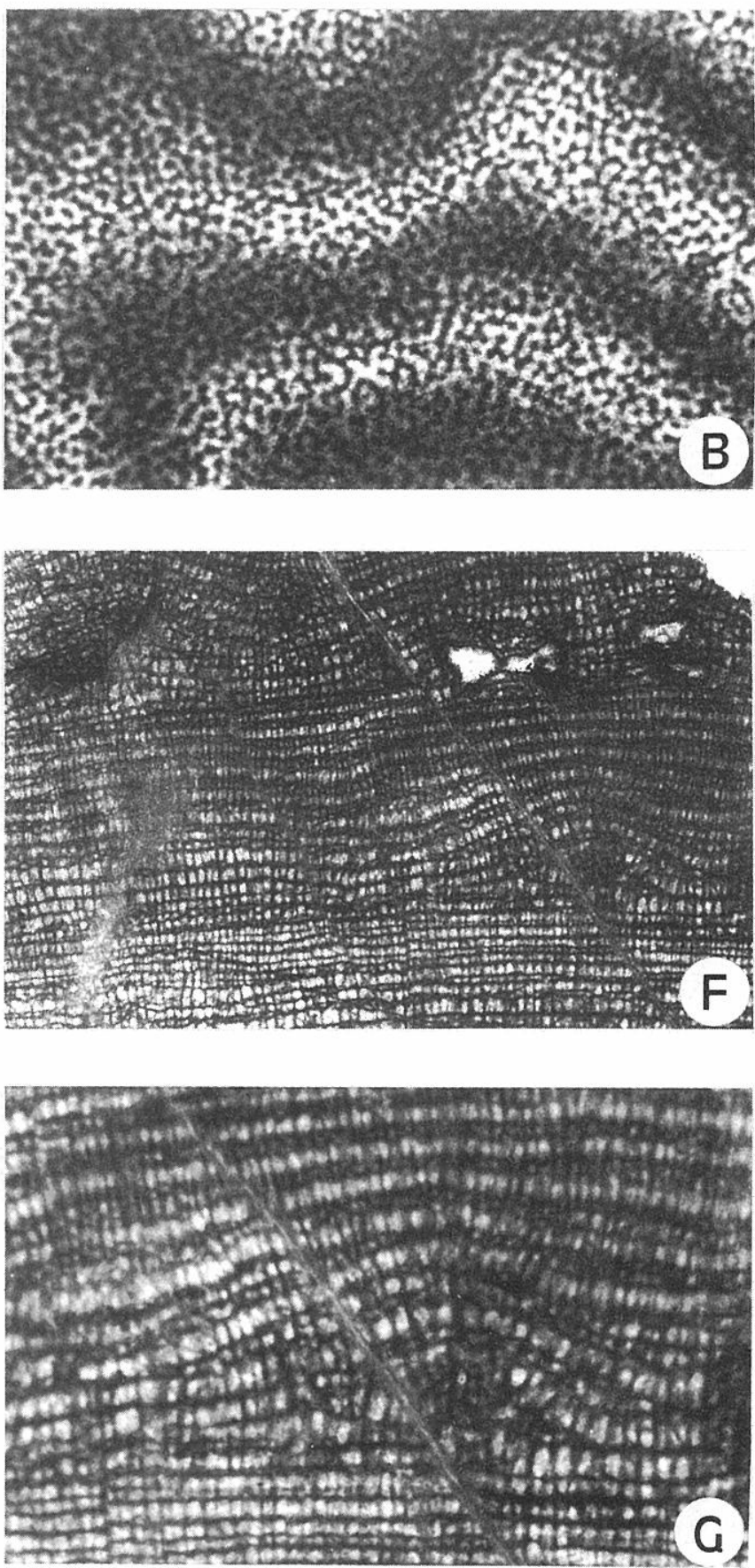

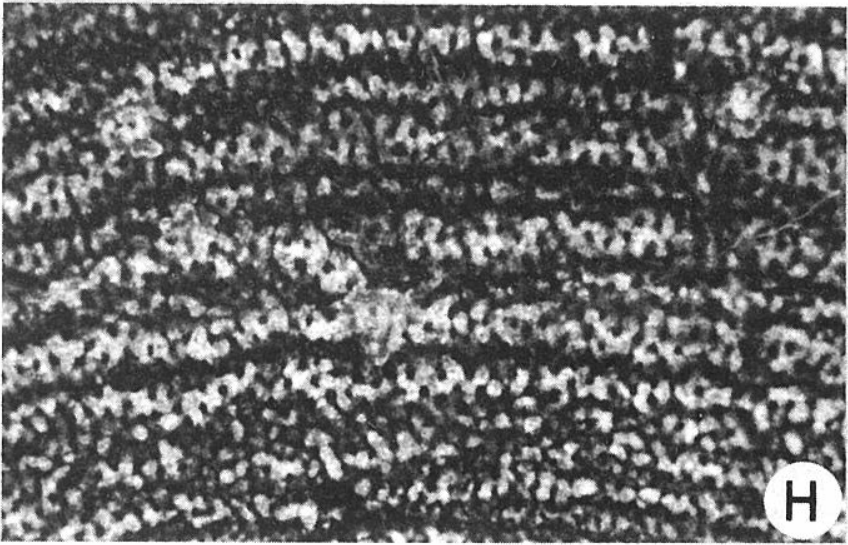



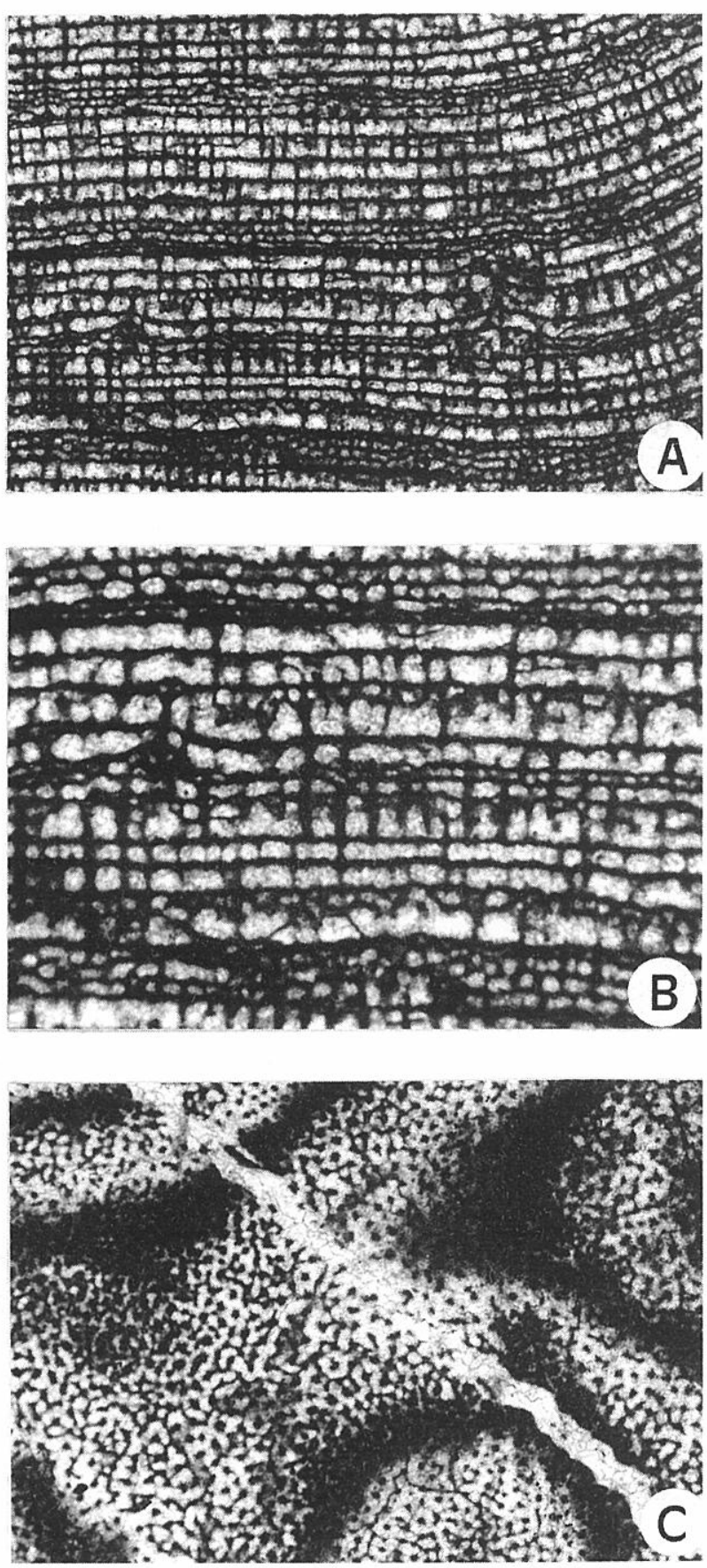

Figura 3. Actinostroma cf. conglomeratum Lecompte, 1951. A, sección vertical del ejemplar DPO 33543 en la que se muestran láminas reemplazadas por alveolos agrupados en una o mas hileras. x 5 . B, detalle de la sección anterior. X 10. C, sección tangencial del mismo ejemplar en la que se observa una malla hexactinélida. x 5 .

República Checa, Marruecos, antigua Unión Soviética, Afganistán y S de China: Devónico Medio; Alemania: Devónico Superior, Frasniense inferior.

Actinostroma geminatum Lecompte, 1951 (Fig. 2F-H) está representada por un único ejemplar, con morfología hemisférica y latilaminación visible. Este espécimen posee los caracteres del género Actinostroma, principalmente por lo que se refiere a la presencia de la malla hexactinélida característica, en corte tangencial, y de pilares que en su mayor parte atraviesan más de un espacio interlaminar. Lecompte (1951) sólo figura una sección vertical del holotipo, por lo que no existe ninguna ilustración de un corte tangencial. El ejemplar estudiado corresponde bien a Actinostroma geminatum, tanto por su estructura general como por la densidad de los elementos esqueléticos, aunque las láminas del ejemplar de la Formación Santa Lucía son algo más delgadas. Esta especie, en particular, tiene un aspecto muy típico dado que presenta agrupaciones de dos o tres láminas separadas por espacios interlaminares más amplios. A veces, las agrupaciones tripartitas le confieren un aspecto celular producido por la interferencia de las láminas irregulares y de los pilares. El espécimen de la Cordillera Cantábrica presenta, como media en $5 \mathrm{~mm}$, unas 23-24 láminas en zonas en donde hay menor número de agrupaciones y 25 a 27 láminas en aquellos lugares en los que éstas se hallan más densamente agrupadas. Su espesor varía de 0,015 a $0,05 \mathrm{~mm}$. Los pilares varían de unas zonas en las que están más separados, siendo la media en $5 \mathrm{~mm}$ de unos 22, a otras en donde se disponen más apretadamente, contándose 25 a 30 pilares, con un espesor muy variable de 0,04 a 0,10 mm. El único ejemplar del que se dispone procede de la localidad de Adrados (ADR-1) y ha sido recolectado en la parte inferior de la Formación Santa Lucía. Esta especie posee una distribución geográfica restringida dentro del Dominio del Viejo Mundo. Aparece en España: Devónico Inferior, Emsiense superior; Bélgica: Devónico Medio, Eifeliense inferior y en Polonia: Devónico Medio, Givetiense.

Actinostroma cf. conglomeratum Lecompte, 1951 (Fig. 3A-C) está representada por 6 ejemplares, en su mayoría fragmentos, con morfologías tabulares a hemisféricas, a veces alcanzando tallas de $50 \mathrm{~cm}$ de largo y $25 \mathrm{~cm}$ de altura; latilaminación visible en alguno de ellos. Los caracteres observados en estos ejemplares corresponden a los del género Actinostroma, y son comparables a los de la especie Actinostroma conglomeratum, por lo que respecta a la estructura general, con láminas onduladas, pero siguiendo una trayectoria rectilínea. A ciertos niveles, las láminas se hallan reemplazadas por alveolos irregulares agrupados en una o dos hileras. A veces, varias láminas concurren formando una lámina compleja. Sin embargo, se diferencian de la especie-tipo por un menor número de pilares y por la estructura alveolar más regular, observándose, a veces, más de dos filas de alveolos. Presentan, por término medio, 18 a 21 láminas en $5 \mathrm{~mm}$, de 0,04 a $0,10 \mathrm{~mm}$ de espesor, y 12 a 14 pilares, de 0,05 a $0,10 \mathrm{~mm}$ de espesor. Estos ejemplares proceden de la parte superior de la Formación Santa Lucía, en la localidad de El Millar (MI-5, MI-6 y MI-7). La distribución geográfica de esta especie está restringida en el Dominio del Viejo Mundo a España (Devónico Inferior, Emsiense superior), Bélgica (Devónico Medio, Eifeliense inferior) y a China (Devónico Medio, Givetiense). 


\section{CONCLUSIONES}

A partir del estudio de las especies analizadas en este trabajo se pueden obtener, por un lado, una serie de conclusiones preliminares de carácter regional relacionadas con el tipo de facies con el que se encuentran asociadas y, por otro, de carácter general con respecto a la distribución paleogeográfica.

Las especies Actinostroma stellulatum y $A$. verrucosum se encuentran tanto en sucesiones de la Formación Santa Lucía con características propias de facies externas (La Vega de los Viejos y Argovejo) como en sucesiones con facies intermedias (Aviados y Adrados). Por el contrario, A. conglomeratum está restringida exclusivamente a las facies externas (El Millar) y A. geminatum aparece solamente en sucesiones con facies intermedias (Adrados).

Las especies de Actinostroma estudiadas en el Devónico Inferior (Emsiense superior) de la Cordillera Cantábrica poseen una distribución estratigráfica y geográfica mucho más amplia a nivel mundial. Desde el punto de vista estratigráfico, estas especies, çon la excepción de A. stellulatum, se encuentran en otras áreas desde el Eifeliense inferior hasta el Frasniense inferior; por lo tanto, las especies A. verrucosum, A. geminatum y A. conglomeratum parecen haber tenido su origen en la Cordillera Cantábrica. Una especie afín a A. stellulatum ha sido citada por Webby y Zhen (1997) en el Emsiense superior de Australia. La distribución geográfica de las especies indicadas muestra claras relaciones, desde el Devónico Inferior (Emsiemse superior) al Devónico Superior (Frasniense inferior), entre la Cordillera Cantábrica y otras cuencas del Dominio del Viejo Mundo situadas en el margen $\mathrm{O}$ del Océano Prototético (República Checa, Francia, Inglaterra, Bélgica, Alemania, Polonia y antigua Unión Soviética) y emplazadas en el margen S del mismo (Marruecos, Austria, Afganistán, S de China y Australia).

\section{AGRADECIMIENTOS}

La autora desea agradecer al Profesor C.W. Stock (Departamento de Geología, Universidad de Alabama) que con sus comentarios y sugerencias ha permitido ultimar este trabajo. Así mismo agradece al Dr. B. Mistiaen (Facutad Libre de Ciencias, Lille) por su constante apoyo en el estudio de estromatoporoideos y a los Drs. F. Soto y M. Arbizu (Departamento de Geología, Universidad de Oviedo) por su ayuda en la lectura crítica del manuscrito y elaboración de las láminas, respectivamente. Al Dr. A. Perejón (Departamento de Paleontología, Universidad Complutense de Madrid) por la revisión del manuscrito que ha permitido mejorar este artículo. Este trabajo ha sido parcialmente financiado por el proyecto PB92-1008 de la CICYT y representa una contribución al proyecto del PICG n $\mathrm{n}^{\circ}$ 421:"North Gondwana mid-Palaeozoic bioevent/biogeogra-phy patterns in relation to crustal dynamics".

\section{BIBLIOGRAFÍA}

Bargatzky, A. 1881. Die Stromatoporen des rheinischen Devons. Verhandlungen des Naturhistorischen Vereins der Preussischen Rheinlande und Westfalens, 38, 233304.

Bargatzky, A. 1883. Observations sur les Stromatopores du terrain Dévonien des Asturies. Annales de la Société Géologique du Nord, 9, 126-131.

Barrois, Ch. 1882. Recherches sur les terrains anciens des Asturies et de la Galice. Mémoires de la Société Géologique du Nord, 2, 1-630.

Brouwer, S.A. 1964. Deux faciès dans le Dévonien des Montagnes Cantabriques Méridionales. Breviora Geologica Asturica, 8(1-4), 3-10.

Flügel, E. 1959. Die Gattung Actinostroma Nicholson und ihre Arten (Stromatoporoidea). Annalen des Naturhistorischen Museums in Wien, 63, 90-273.

Goldfuss, A. 1826. Petrefacta Germaniae. Arnz \& Co., 1, 176.

Lecompte, M. 1951. Les Stromatoporoïdes du Dévonien Moyen et Supérieur du Bassin de Dinant. Première Partie. Mémoires du Institut Royal des Sciences Naturelles de Belgique, 116, 1-125.

Lesovaya, A.I. 1970. Stromatoporoidei devona Zeravshanskogo khrebta. In: Biostratigrafiya osadochnykh obrazovanii Uzbekistana (Ed. T.S. Shayakubov). Ministerstvo Geologii Uz-SSR Tashkentgeologiya, 9, 74-109.

Mallett, C.W. 1971. The stromatoporoid genera Actinostroma Nicholson and Nexililamina gen. nov. from the Devonian Broken River Formation, North Queensland. Proceedings of the Royal Society Victoria, 84, 235-244.

Méndez-Bedia, I. 1976. Biofacies y Litofacies de la Formación Moniello-Santa Lucía (Devónico de la Cordillera Cantábrica, NW de España). Trabajos de Geología, Universidad de Oviedo, 9, 1-93.

Méndez-Bedia, I. 1984. Primera nota sobre los Estromatopóridos de la Formación Moniello (Devónico de la Cordillera Cantábrica, NW de España). Trabajos de Geología, Universidad de Oviedo, 14, 151-159.

Méndez-Bedia, I. and Mistiaen, B. 1997. Genus Stromatoporella Nicholson, 1886 from the Cantabrian Mountains (Santa Lucía Formation, Lower-Middle Devonian, NW Spain). Boletín de la Real Sociedad Española de Historia Natural (Sección Geológica), 91, 343-353.

Méndez-Bedia, I., Soto, F. and Fernández-Martínez, E. 1994. Devonian reef types in the Cantabrian Mountains (NW Spain) and their faunal composition. Courier Forschunginstitut Senckenberg, 172, 161-183.

Nicholson, H.A. 1886a. On some new or imperfectly-known species of Stromatoporoids. Annals and Magazine of Natural History, 17, 225-239.

Nicholson, H.A. 1886b. A monograph of the British Stromatoporoids. Part I. General Introduction. Palaeontographical Society, London, 39, 1-130.

Nicholson, H.A. 1889. A monograph of the British Stromatoporoids. Part II. Description of Species. 
Palaeontographical Society, London, 42, 131-158.

Nicholson, H.A. 1891. A monograph of the British Stromatoporoids. Part III. Description of Species. Palaeontographical Society, London, 44, 159-202.

Sleumer, B.H.G. 1969. Devonian Stromatoporoids of the Cantabrian Mountains (Spain). Leidse Geologische Mededelingen, 44, 1-52.

Soto, F. y Méndez-Bedia, I. 1985. Estudio de una asociación coral rugoso-estromatopórido en el arrecife de Arnao (Fm. Moniello, Asturias, NO de España). Trabajos de Geología, Universidad de Oviedo, 15, 203-209.

Stearn, C.W. 1966. The microstructure of stromatoporoids. Palaeontology, 9, 74-124.

Stearn, C.W. 1980. Classification of the Paleozoic Stromatoporoids. Journal of Paleontology, 54, 881-902.

Stearn, C.W. 1991. A revision of Anostylostroma, Atelodictyon, and related genera (Paleozoic Stromatoporoidea). Journal of Paleontology, 65, 611-622.

Stock, C.W. 1982. Upper Devonian (Frasnian) Stromatoporoidea of North-Central Iowa: Mason City
Member of the Shell Rock Formation. Journal of Paleontology, 56, 654-679.

Truyols, J., Arbizu, M.A., García-Alcalde, J.L., García López, S., Méndez-Bedia, I., Soto, F. and TruyolsMassoni, M. 1990. The Asturian-Leonese Domain (Cantabrian Zone). In: Pre-Mesozoic Geology of Iberia (Eds. R.D. Dallmeyer and E. Martínez García). Springer Verlag, part II, Cantabrian and Palentian Zones, 2. Stratigraphy, 2.2, 10-19.

Ünsalaner, C. 1951. Some Upper Devonian corals and stromatoporoids from South Anatolia. Bulletin Geological Survey of Turkey, 3(1), 131-146.

Webby, B.D. and Zhen, Y.Y. 1997. Silurian and Devonian clathrodictyids and other stromatoporoids from the Broken River region, north Queensland. Alcheringa, 21, $1-56$.

Yavorsky, V.I. 1931. Nekotorye devonskie Stromatoporoidea iz okrain Kuznetskogo basseina, Urala i drugikh mest. Izvestiya vsesoyuznogo geologo-razvedochnogo obyedineniya, 50, 1387-1415.

Manuscrito recibido: 12 de enero, 1998 Manuscrito aceptado: 6 de septiembre, 1998 